\title{
Delay Pressure Detection Method to Eliminate Pump Pressure Interference on the Downhole Mud Pressure Signals
}

\author{
Yue Shen, ${ }^{1}$ Ling-Tan Zhang, ${ }^{1}$ Shi-Li Cui, ${ }^{1}$ Li-Min Sheng, ${ }^{2}$ Lin $\mathrm{Li}^{2}{ }^{2}$ and Yi-Nao Su${ }^{2}$ \\ ${ }^{1}$ School of Science, China University of Petroleum, Qingdao 266580, China \\ ${ }^{2}$ Drilling Technology Research Institute, CNPC, Beijing 100195, China \\ Correspondence should be addressed to Yue Shen; sheny1961@aliyun.com
}

Received 12 September 2013; Accepted 12 October 2013

Academic Editor: Teen-Hang Meen

Copyright (c) 2013 Yue Shen et al. This is an open access article distributed under the Creative Commons Attribution License, which permits unrestricted use, distribution, and reproduction in any medium, provided the original work is properly cited.

\begin{abstract}
The feasibility of applying delay pressure detection method to eliminate mud pump pressure interference on the downhole mud pressure signals is studied. Two pressure sensors mounted on the mud pipe in some distance apart are provided to detect the downhole mud continuous pressure wave signals on the surface according to the delayed time produced by mud pressure wave transmitting between the two sensors. A mathematical model of delay pressure detection is built by analysis of transmission path between mud pump pressure interference and downhole mud pressure signals. Considering pressure signal transmission characteristics of the mud pipe, a mathematical model of ideal low-pass filter for limited frequency band signal is introduced to study the pole frequency impact on the signal reconstruction and the constraints of pressure sensor distance are obtained by pole frequencies analysis. Theoretical calculation and numerical simulation show that the method can effectively eliminate mud pump pressure interference and the downhole mud continuous pressure wave signals can be reconstructed successfully with a significant improvement in signal-to-noise ratio (SNR) in the condition of satisfying the constraints of pressure sensor distance.
\end{abstract}

\section{Introduction}

In measurement while drilling (MWD), various downhole signals will be transmitted to the surface in real time for instructing the drilling operation. One of the most common methods of transmitting the measured downhole information to the surface is through mud pressure pulses produced by mechanical modulation of a mud siren in MWD tools and transmitted at acoustic speed in the mud flow. The mud siren generates mud continuous pressure wave signals with complex modulation methods to produce higher data rates. When transmitting the mud pressure signals, there will be a lot of pressure noise and interference, among which the mud pressure fluctuation generated by the mud pump contributes to the largest influence. The mud pump pressure interference is related to the pump stoke rate which includes fundamental component and harmonic component. When the mud pump is in imbalance operation mode caused by sealing problem or in abnormal working status, some higher harmonic amplitude will become very large. Although the pressure dampers are equipped on mud pump pipe, the pressure fluctuation generated by mud pump reaches or exceeds the downhole signal strength detected in the stand pipe [1]. These higher harmonics will enter the frequency band of mud pressure signal and thereby create great interference that cannot be eliminated by conventional signal processing method, leading to the great decrease of signal-to-noise ratio (SNR) of signal and affecting extraction of the MWD signals. Many studies had been done to eliminate the pump interference. Marsh and others proposed the matched filter method which treated mud pump interference as random noise and calculated the autocorrelation coefficient to eliminate the mud pump pressure interference [2]. However, the pump interference is a kind of system interference rather than random noise, so the conclusions of the method needed further discussion. Brandon and others proposed an adaptive compensation method which uses extracted interference component in the signal and automatically adjusts strength of the interference component to eliminate the pump pressure interference impact on the signal [3], but the effect was 


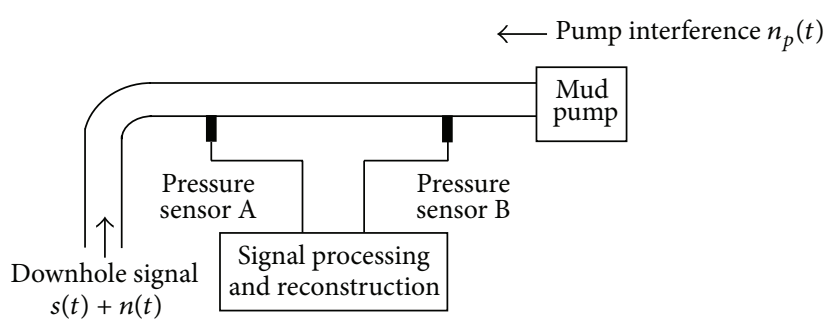

FIGURE 1: Mud pressure detection system.

limited. Some literatures [4-7] introduced the delay pressure detection technique and built a mathematical model, being fitted to the single-frequency signal with pressure sensors distance of quarter signal wavelength, for eliminating the mud pump pressure interference. Because components of many frequencies are contained in mud continuous pressure wave signals, the mathematical model presented in those literatures cannot be applied in reconstruction of actual mud continuous pressure wave signals. Based on transmission path analysis of mud pump pressure interference and downhole mud pressure signals, the authors established the mathematical model in time domain for processing mud continuous pressure wave signals according to the fundamental mathematical principle of delay pressure detection method and then studied the reconstruction method of mud continuous wave signals in both time domain and frequency domain and constraints of the distance between pressure sensors.

\section{Mathematical Model of Delay Pressure Detection}

The delay pressure detection method uses two pressure sensors being some distance apart on the mud pipe to detect and process the mud pressure signal; Figure 1 shows the schematic figure of mud pressure signal detection system. Two pressure sensors, $\mathrm{A}$ and $\mathrm{B}$, having distance $L_{0}$ between each other, are equipped in a straight pipe between wellhead and mud pump. The pressure signals received by two sensors contain downhole signal (mud pressure signal) $s(t)$, downhole random noise $n(t)$, and mud pump pressure interference $n_{p}(t)$. The transmission direction of pump pressure interference is opposite to that of downhole signal. Suppose that the propagation velocity of the mud pressure wave is $c_{0}$ and the pressure wave transmission time between sensors $\mathrm{A}$ and $\mathrm{B}$ is $\tau_{0}=L_{0} / c_{0}$.

Considering the mud pipe between sensors A and B as a linear system, its frequency response can be described as

$$
H(j \omega)=|H(j \omega)| \cdot e^{-j \omega \tau_{0}},
$$

where $|H(j \omega)|$ is modulus of frequency domain transfer function of the mud pipe between pressure sensors A and B.

Suppose that $h(t)$ is unit impulse response of the linear system $H(j \omega)$. When the signal is being transmitted through the linear system [8], signals received by the pressure sensors $A$ and $B$ can be expressed as

$$
\begin{gathered}
p_{\mathrm{A}}(t)=s(t)+n(t)+h(t) * n_{p}(t), \\
p_{\mathrm{B}}(t)=h(t) *[s(t)+n(t)]+n_{p}(t) .
\end{gathered}
$$

We can get convolution of $h(t)$ with $p_{\mathrm{B}}(t)$ as

$$
h(t) * p_{\mathrm{B}}(t)=h(t) * h(t) *[s(t)+n(t)]+h(t) * n_{p}(t) .
$$

Equation (3) means that $p_{\mathrm{B}}(t)$ is transmitted through a linear system with unit impulse response $h(t)$ again. Because $h(t)$ contains delayed time $\tau_{0}=L / c_{0}$, the physical meaning of (3) is that $p_{\mathrm{B}}(t)$ will be detected after delayed time $\tau_{0}$.

Subtracting the formula in (3) from $p_{\mathrm{A}}(t)$, we can get the delay pressure detecting signal as follows:

$$
\begin{aligned}
p_{\mathrm{A}}(t)-h(t) * p_{\mathrm{B}}(t)= & s(t)+n(t) \\
& -h(t) * h(t) *[s(t)+n(t)] .
\end{aligned}
$$

In (4), the pump pressure interference item $n_{p}(t)$ has been eliminated.

After Fourier transform of the formula in (4), we can get spectral density function of the downhole signal as

$$
S(j \omega)+N(j \omega)=H^{\prime}(j \omega)\left[P_{\mathrm{A}}(j \omega)-P_{\mathrm{B}}(j \omega) \cdot H(j \omega)\right],
$$

where $H^{\prime}(j \omega)=1 /(1-H(j \omega) \cdot H(j \omega))$ can be applied to reconstruct the downhole signal.

\section{Signal Reconstruction Based on Time-Domain Differential Equation}

According to (5), the time-domain solution of the system frequency response can be described as the reconstruction of the downhole signal after the delay pressure detecting signal $p_{\mathrm{A}}(t)-h(t) * p_{\mathrm{B}}(t)$ is passed through a signal recovering system with frequency transfer function $H^{\prime}(j \omega)$.

Considering that the maximum frequency of mud continuous pressure wave signal in transmission will be dozens of hertz $(\mathrm{Hz})$, the signal frequency is lower and limited. In limited frequency band, the signal attenuation in amplitude will keep unchangeable when mud continuous pressure wave signal passes the straight pipe between pressure sensors A and $B$, so the pipe can be seen as an undistorted transmission system and regarded as an ideal low-pass filter. The frequency domain transfer function of the system can be described as

$$
H(j \omega)=a G(\omega) e^{-j \omega \tau_{0}},
$$

where $a$ is the signal attenuation coefficient and $G(\omega)$ is unit gate function with $\omega_{b}$ as unilateral bandwidth. According to the unit impulse response of ideal low-pass filter [9], the unit impulse response of system $H(j \omega)$ can be described as

$$
h(t)=\frac{a \omega_{b}}{\pi} \cdot \frac{\sin \left[\omega_{b}\left(t-\tau_{0}\right)\right]}{\omega_{b}\left(t-\tau_{0}\right)}=\frac{a \omega_{b}}{\pi} \operatorname{Sinc}\left[\omega_{b}\left(t-\tau_{0}\right)\right] .
$$


After reciprocal transformation of $H^{\prime}(j \omega)$, we can get

$$
H_{1}(j \omega)=\frac{1}{H^{\prime}(j \omega)}=\frac{Y_{1}(j \omega)}{X_{1}(j \omega)}=1-H(j \omega) \cdot H(j \omega) .
$$

Then

$$
Y_{1}(j \omega)=X_{1}(j \omega)-H(j \omega) \cdot H(j \omega) \cdot X_{1}(j \omega) .
$$

Because transfer functions of $H_{1}(j \omega)$ and $H^{\prime}(j \omega)$ are reciprocal, so their input and output functions are inverse of each other.

Substituting input function $x(t)$ and output function $y(t)$ of $H^{\prime}(j \omega)$ for output function $y_{1}(t)$ and input function $x_{1}(t)$ of $H_{1}(j \omega)$ in (9), there will be

$$
x(t)=y(t)-\left(\frac{a \omega_{b}}{\pi}\right)^{2} \operatorname{Sinc}\left(\omega_{b} t\right) * \operatorname{Sinc}\left(\omega_{b} t\right) * y\left(t-2 \tau_{0}\right) .
$$

Thus, the time-domain solution of the output function of $H^{\prime}(j \omega)$ can be built as

$$
\begin{aligned}
y(t)= & x(t)+\left(\frac{a \omega_{b}}{\pi}\right)^{2} \operatorname{Sinc}\left(\omega_{b} t\right) * \operatorname{Sinc}\left(\omega_{b} t\right) \\
& * y\left(t-2 \tau_{0}\right),
\end{aligned}
$$

where $x(t)$ is delay pressure detecting signal and can be expressed as $x(t)=p_{\mathrm{A}}(t)-h(t) * p_{\mathrm{B}}(t)$ and $y(t)=s(t)+n(t)$ is reconstructed downhole signal.

Converting the continuous-time system to Z-system of discrete-time and setting $z=e^{j \omega T_{s}}, k=2 \tau_{0} / T_{s}$, and $t=N T_{s}$, we can get the Z-transform of $H^{\prime}(j \omega)$ as

$$
H^{\prime}(z)=\frac{1}{1-|H(z)|^{2} z^{-k}}
$$

where $T_{s}$ is the sampling period and $N$ is the number of sample sequences.

According to digital filter theory, $H^{\prime}(z)$ is a $k$-order infinite impulse response (IIR) filter system [10] and its frequency domain response, being similar to the low-pass filter with sharp cut-off characteristic, strengthens with $k$. When $H(z)$ is an ideal low-pass transmission, the output of $H^{\prime}(z)$ is a differential equation and can be expressed as

$$
\begin{aligned}
y(N)= & x(N)+\left(\frac{a \omega_{\mathrm{b}}}{\pi}\right)^{2} \operatorname{Sinc}\left(\omega_{\mathrm{b}} N\right) * \operatorname{Sinc}\left(\omega_{\mathrm{b}} N\right) \\
& * y(N-k) .
\end{aligned}
$$

Equations (13) and (11) have the same structure, so the essence of signal reconstruction process in time domain is to make the delay pressure detecting signal pass through a closed-loop delay feedback system with recursive structure.

\section{Signal Reconstruction and Pole Frequency Analysis Based on Inverse Fourier Transform}

The straight pipe between pressure sensors A and B will cause pressure signal attenuation. According to the transmission characteristics of mud pressure wave [11], the attenuation coefficient of pressure signal or the amplitude ratio of mud pipe can be described as

$$
a=e^{-L_{0} / D}
$$

with

$$
\begin{gathered}
D=\frac{d}{2} \sqrt{\frac{K_{l}}{\pi f \mu\left[1+\psi\left(\left(K_{l} d\right) / E e\right)+\beta_{g}\left(\left(K_{l} / K_{g}\right)-1\right)+\beta_{s}\left(\left(K_{l} / K_{s}\right)-1\right)\right]}}, \\
\psi=\frac{1}{1+(e / d)}\left[\left(1-\frac{\sigma}{2}\right)+2 \frac{e}{d}(1+\sigma)\left(1+\frac{e}{d}\right)\right]
\end{gathered}
$$

where $L_{0}$ is the pipe length between pressure sensors $A$ and $\mathrm{B}, D$ is the attenuation index, $\beta_{g}$ is the volume fraction of gas in mud, $\beta_{s}$ is the volume fraction of solids in mud, $K_{g}$ is the bulk modulus of gas in mud, $K_{l}$ is the bulk modulus of liquid in mud, $K_{s}$ is the bulk modulus of solid in mud, $E$ is the bulk modulus of the mud pipe, $d$ is the internal diameter of the mud pipe, $e$ is the wall thickness of the mud pipe, $\sigma$ is the Poisson's ratio of the mud pipe, $\mu$ is the kinematic viscosity of mud, and $f$ is signal frequency.

Because the mud pipe forms an ideal low-pass filter in the limited band $\omega<\omega_{b}$, (5) can be transformed into

$$
S(j \omega)+N(j \omega)=\frac{\left[P_{\mathrm{A}}(j \omega)-P_{\mathrm{B}}(j \omega) \cdot H(j \omega)\right]}{1-a^{2} G^{2}(\omega) \cdot e^{-j 2 \omega \tau_{0}}} .
$$

After inverse Fourier transform of (16), we can get the time-domain solution of (16):

$$
\begin{aligned}
y(t) & =s(t)+n(t) \\
& =\frac{1}{2 \pi} \int_{-\infty}^{+\infty} \frac{\left[P_{\mathrm{A}}(j \omega)-P_{\mathrm{B}}(j \omega) \cdot H(j \omega)\right]}{1-a^{2} G^{2}(\omega) \cdot e^{-j 2 \omega \tau_{0}}} e^{j \omega t} d \omega .
\end{aligned}
$$

Suppose that the mud is water-based mud. The computational conditions are listed as follows [12]: internal diameter of the mud pipe is $108.6 \mathrm{~mm}$, wall thickness of the mud pipe is $9.2 \mathrm{~mm}$, the mud kinematic viscosity is $20 \mathrm{mPa} \cdot \mathrm{s}$, the pipe Poisson's ratio is 0.3 , volume fraction of gas in mud is $0.5 \%$, volume fraction of solid in mud is $15 \%$, the mud pipe bulk modulus is $210 \mathrm{GPa}$, and bulk modulus of water in mud is $2.04 \mathrm{GPa}$, bulk modulus of solid in mud is $16.2 \mathrm{GPa}$. If signal frequency of mud continuous pressure wave is $f<f_{b}=$ $40 \mathrm{~Hz}$, when the distance between pressure sensors $\mathrm{A}$ and $\mathrm{B}$ is less than $18 \mathrm{~m}$, the pressure signal attenuation coefficient will be $a>0.988$ by numerical calculation. This means that transmission loss of mud pressure wave signal is very small and the attenuation coefficient will be close to 1 when the two sensors are nearer to each other.

When $a=1$, the transfer function of downhole signal reconstruction system can be expressed as

$$
\begin{aligned}
\left.H^{\prime}(j \omega)\right|_{\omega<\omega_{\mathrm{b}}} & =\frac{1}{1-G^{2}(\omega) \cdot e^{-j 2 \omega \tau_{0}}} \\
& =\frac{1}{1-\cos \left(2 \omega \tau_{0}\right)+j \sin \left(2 \omega \tau_{0}\right)} .
\end{aligned}
$$




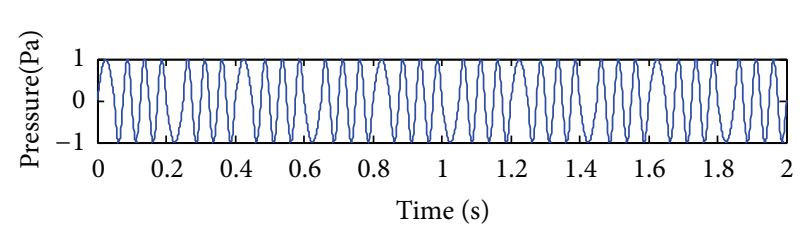

(a) Mud pressure DPSK signal without pump interference

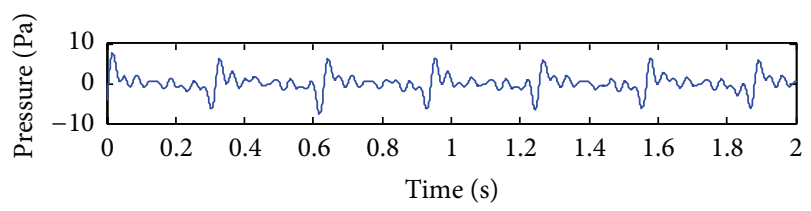

(c) Mud pressure DPSK signal mixed with pump interference

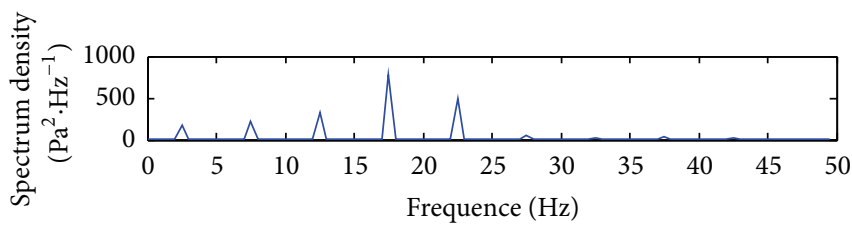

(b) Frequency spectrum of mud pressure DPSK signal

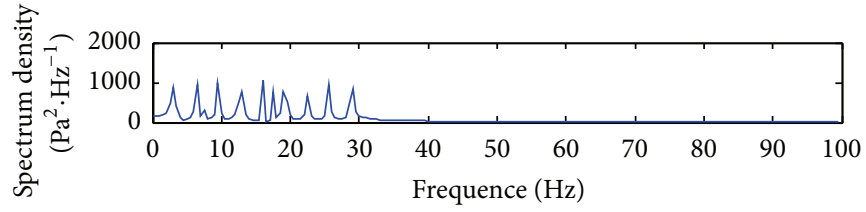

(d) Frequency spectrum of mud pressure DPSK signal mixed with pump interference

FIGURE 2: Mud pressure DPSK signal mixed with pump interference.

By analyzing (18), there will be generated pole in the condition of $2 \omega \tau_{0}=2 m \pi(m=1,2,3, \ldots)$ and the corresponding pole frequency is $f_{0}=m / 2 \tau_{0}$.

If the maximum frequency of mud pressure signal spectrum is $f_{\max }$, there is $f_{\max }<f_{b}$. When the corresponding pole frequency falls into the passband of ideal low-pass filter, the pole frequency will be very likely to enter signal spectrum and generate great interference in the reconstruction of downhole signal. To avoid such situation, all the pole frequency values should be greater than the passband frequency of ideal lowpass filter. That is, $f_{0}=m / 2 \tau_{0}>f_{b}$.

Suppose that $m=1$ and $\tau_{0}<1 / 2 f_{b}$; we can get the constraints of distance between pressure sensors:

$$
L_{0}=\tau_{0} c_{0}<\frac{c_{0}}{2 f_{b}} .
$$

Propagation velocity of the mud pressure wave in the mud pipe can be calculated according to the literature [13]. Take the mud pressure DPSK (differential phase shift keying) signal with carrier wave frequency of $24 \mathrm{~Hz}$ for example, the maximum frequency of signal spectrum is $36 \mathrm{~Hz}$. When $f_{b}=$ $40 \mathrm{~Hz}$, we have $\tau_{0}<1 / 80 \mathrm{~s}$. Furthermore, if the mud pressure wave velocity is $c_{0}=1280 \mathrm{~m} / \mathrm{s}$, the corresponding distance between pressure sensors is $L_{0}=\tau_{0} c_{0}<16 \mathrm{~m}$.

\section{Numerical Simulation of Signal Reconstruction}

The numerical simulation takes mud pressure DPSK signal as an example. According to the mathematical model of mud pressure DPSK signal [14], the signal can be formulated as $s(t)=A_{c} \sin \left[2 \pi f_{c} t-f(t)\right]$. In the formula, carrier frequency is $f_{c}=20 \mathrm{~Hz}$, signal amplitude is $A_{c}=1 \mathrm{~Pa}$, and data code is $C=\left[\begin{array}{llllllllll}1 & 1 & 1 & 1 & 1 & 1 & 1 & 1 & 1 & 1\end{array}\right]$. By analyzing the power spectral of mud pressure DPSK signal, the maximum frequency of signal spectrum is $f_{\max }=30 \mathrm{~Hz}$ and the signal power is $P_{s}=\left(A_{c} / \sqrt{2}\right)^{2}=0.5 \mathrm{~Pa}^{2}$. Mud pump interference simulates multifrequency pressure pulsation generated by triplex pump with pump impulse rate $64 \mathrm{r} / \mathrm{min}$, and the fundamental wave frequency is $f_{1}=3 \times 64 / 60=3.2 \mathrm{~Hz}$ with harmonic orders 2 to 9. Therefore, the frequency changing range of pump interference is from $3.2 \mathrm{~Hz}$ to $28.8 \mathrm{~Hz}$. Suppose that the fundamental wave and every harmonic wave amplitude are $A_{i}=1 \mathrm{~Pa}$. The corresponding power density of fundamental wave or every harmonic wave is an impact function $S(f)=\left(A_{i} / \sqrt{2}\right)^{2} \delta\left(f-f_{i}\right)$ and the average power of the pump interference is

$$
\begin{aligned}
P_{n} & =\int_{-\infty}^{+\infty} S(f) d f=\int_{-\infty}^{+\infty}\left(\frac{A_{i}}{\sqrt{2}}\right)^{2} \delta\left(f-f_{i}\right) d f \\
& =\sum_{i=1}^{9} \frac{A_{i}^{2}}{2}=4.52 \mathrm{~Pa}^{2} .
\end{aligned}
$$

Therefore, the SNR of signal mixed with the pump interference is $P_{\mathrm{s}} / P_{n}=0.11$ when downhole noise is set to $n(t)=0$.

Figure 2 shows the signal waveform and the signal spectrum mixed with mud pump interference. It can be seen that the mud pressure DPSK signal is completely submerged in the pump interference in time domain and the signal spectrum is completely covered by mud pump interference frequencies.

Suppose that the signal acts on the $H^{\prime}(j \omega)$ at $t=0, H^{\prime}(j \omega)$ has zero state response only, and the system output before $t=$ 0 is $y\left(0^{-}\right)=0$. Simulation result of the reconstructed signal by MATLAB programming is shown in Figure 3. It can be seen that the mud pump interference is eliminated after delay pressure detection from Figure 3(a); the reconstructed signal in Figures 3(b) and 3(c) are consistent with the mud pressure DPSK signal in Figure 2(a). In Figure 3(b), the numerical calculation result shows that the SNR of reconstructed mud pressure DPSK signal under condition of $\tau_{0}=3.91 \mathrm{~ms}$ is 72.4 , which is about 657 times higher than that of existing pump interference. Numerical calculation and analysis show that the SNR of reconstructed mud pressure DPSK signal will be affected by the delayed time $\tau_{0}$ in time domain and the influence is listed in Table 1 . The reason is that the set value of $y\left(0^{-}\right)=0$, participating in the recursive computation in (11), will be increased with the delayed time $\tau_{0}$, 


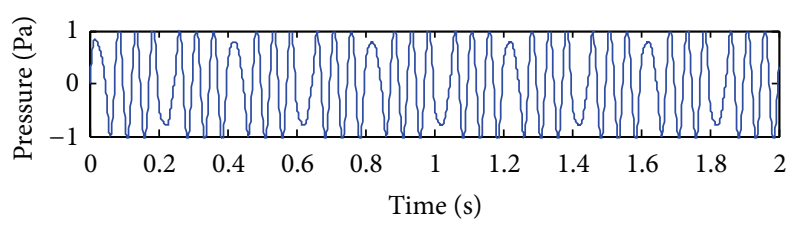

(a) Signal waveform after delay pressure detecting

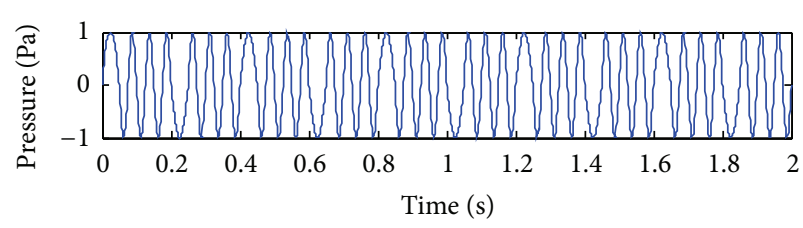

(b) Reconstructed signal of mud pressure DPSK signal with timedomain differential equation

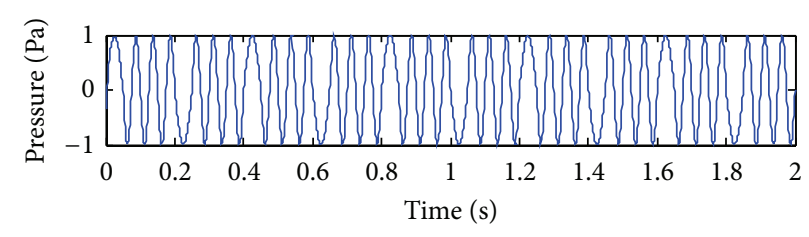

(c) Reconstructed signal of mud pressure DPSK signal based on inverse Fourier transform

FIGURE 3: Reconstruction of mud pressure DPSK signal.

TABLE 1: SNR of reconstructed mud pressure DPSK signal.

\begin{tabular}{lc}
\hline Delayed time $\tau_{0}(\mathrm{~ms})$ & $\begin{array}{c}\text { SNR of reconstructed mud } \\
\text { pressure DPSK signal }\end{array}$ \\
\hline 0.98 & 72.6 \\
1.95 & 72.6 \\
2.93 & 72.5 \\
3.91 & 72.4 \\
4.89 & 72.1 \\
5.86 & 70.5 \\
\hline
\end{tabular}

but the influence is not notable. In Figure 3(c), the reconstructed mud pressure DPSK signal based on inverse Fourier transform method has no distortion in whole waveform and is better than the signal reconstructed by time-domain differential equation method in quality. However, both reconstruction methods can reconstruct downhole signal effectively.

Numerical simulation shows that if downhole noise is added to DPSK signal, the reconstructed signals based on the two reconstruction methods are the linear superposition of DPSK signal and downhole noise, which is consistent with theoretical analysis of (11) and (17).

\section{Conclusions}

(1) Theoretical analysis and numerical simulation show that delay pressure detection method can effectively eliminate mud pump interference and realize reconstruction or recovery of mud continuous pressure wave signals with greater SNR.

(2) To avoid the pole frequency entering into the signals frequency band in signal reconstruction, the distance between pressure sensors should be determined according to the highest signal frequency and the minimum wave velocity.

(3) According to the mathematical principle analysis of delay pressure detection method, it is only applied to eliminate special interference (mud pump pressure interference) whose transmitting direction is opposite to that of the downhole signal. For mud continuous pressure wave signal which is seriously affected by mud pump interference, this method has some inspiration effect on solving the problem of mud pump pressure interference.

\section{Acknowledgments}

This work was supported by the Project of National Natural Science Foundation of China (no. 51274236) and the Project of High-tech Research and Development Program of China (no. 2006AA06A101).

\section{References}

[1] R. Hutin, R. W. Tennent, and S. V. Kashikar, "New mud pulse telemetry techniques for deepwater applications and improved real-time data capabilities," in Proceedings of the SPE/IADC Drilling Conference, vol. 67762 of SPE, pp. 73-82, March 2001.

[2] J. L. Marsh, E. C. Fraser, and A. L. Holt Jr., "Measurementwhile-drilling mud pulse detection process: an investigation of matched filter responses to simulated and real mud pressure pulses," in Proceedings of the Petroleum Industry Applications of Microcomputers, SPE, pp. 119-17787, June 1988.

[3] T. L. Brandon, M. P. Mintchev, and H. Tabler, "Adaptive compensation of the mud pump noise in a measurement-whiledrilling system," SPE Journal, vol. 4, no. 2, pp. 128-133, 1999.

[4] B. A. Montaron, J.-M. D. Hache, and B. Voisin, "Improvements in MWD telemetry: 'the right data at the right time," in Proceedings of the Asia Pacific Oil and Gas Conference, vol. 25356 of SPE, pp. 337-346, February 1993.

[5] C. A. Martin, R. M. Philo, D. P. Decker, and T. M. Burgess, "Innovative advance in MWD," in Proceedings of the Drilling Conference, vol. 27516 of SPE, pp. 787-796, February 1994.

[6] C. Klotz, P. Bond, I. Wasserman, and S. Priegnitz, "A new mud pulse telemetry system for enhanced MWD/LWD applications," in IADC/SPE Drilling Conference, vol. 112683 of SPE, pp. 949953, Orlando, Fla, USA, March 2008.

[7] M. R. Foster and B. J. Patton, "Apparatus for improving signalto-noise ratio in logging-while-drilling system," U. S. Patent, 3742443, 1973. 
[8] X. J. Sun and B. J. Wang, Principles of Communication, Publishing House of Electronics Industry, Beijing, China, 2001.

[9] D. Z. Wu, Signals and Linear Net Analysis, Higher Education Press, Beijing, China, 1980.

[10] Z. Y. Wu, Digital Signals Processing, Higher Education Press, Beijing, China, 2004.

[11] Y. Shen, J. Zhu, Y. Su, L. Sheng, and L. Li, "Transmission characteristics of the drilling fluid pressure quadrature phase shift keying signal along a directional wellbore," Acta Petrolei Sinica, vol. 32, no. 2, pp. 340-345, 2011.

[12] Y. Shen, C. Li, J. Zhu, Y.-N. Su, and L. Li, "Numerical modeling and characteristics analysis of drilling fluid pressure MPSK signals," Journal of China University of Petroleum, vol. 34, no. 5, pp. 77-83, 2010.

[13] X. S. Liu and Y. N. Su, "Study on transmission velocity of mud pulse signal," Petroleum Drilling Techniques, vol. 28, no. 5, pp. 24-26, 2000.

[14] Y. Shen, Y. N. Su, G. S. Li et al., "Transmission characteristics of DPSK mud pressure signals in a straight well," Petroleum Science and Technology, vol. 29, no. 12, pp. 1249-1256, 2011. 


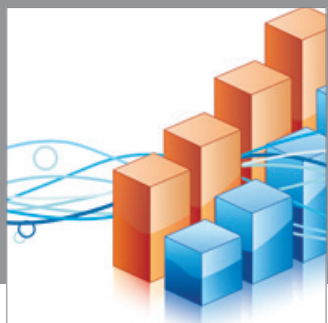

Advances in

Operations Research

mansans

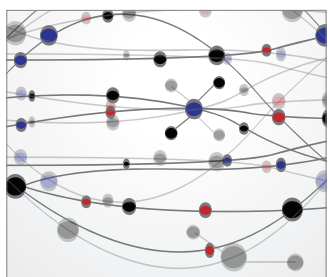

The Scientific World Journal
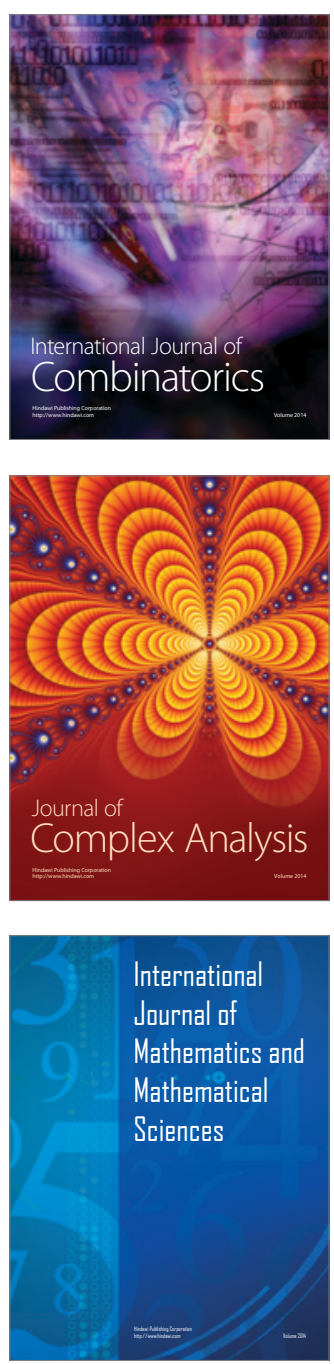
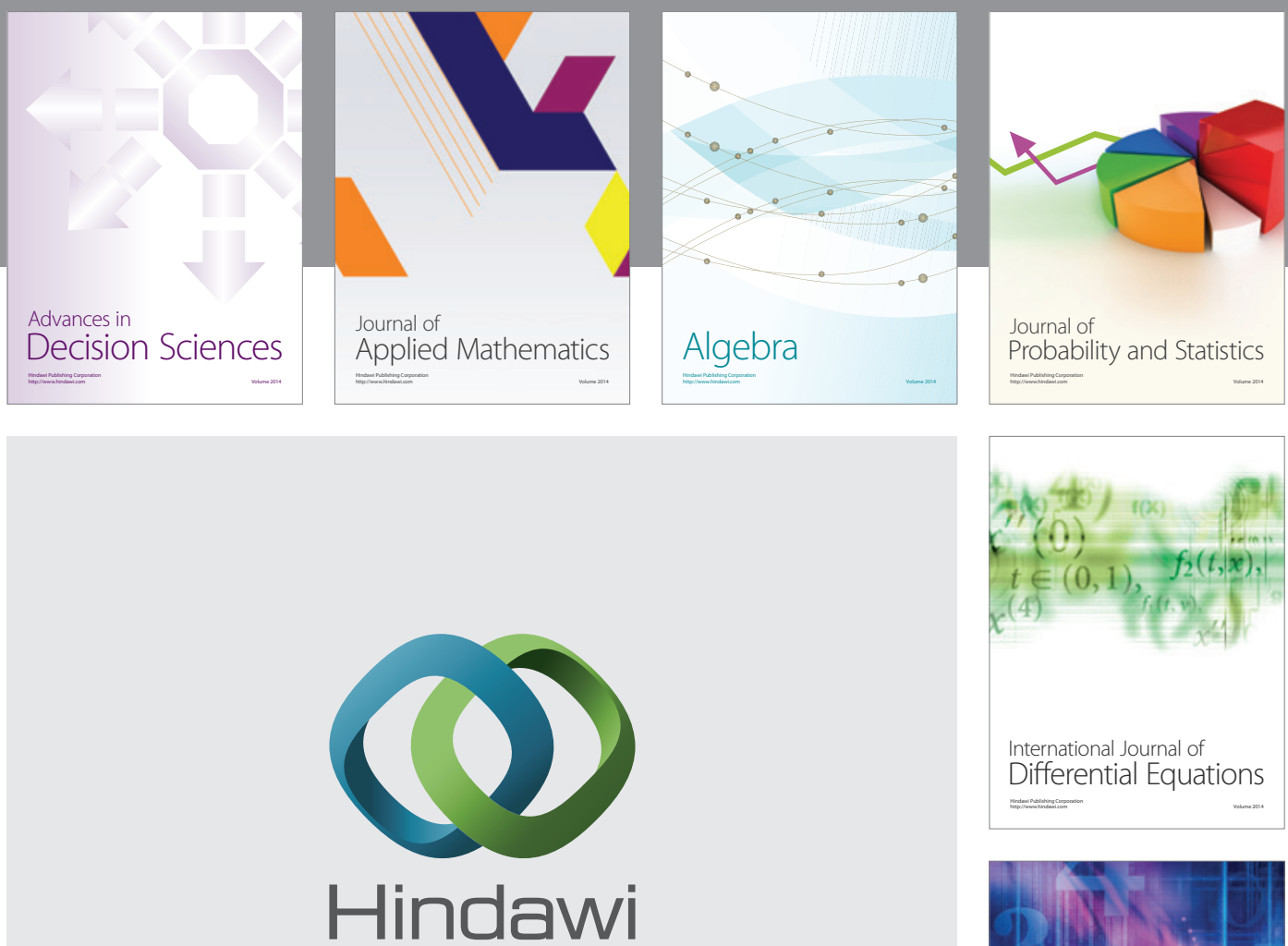

Submit your manuscripts at http://www.hindawi.com
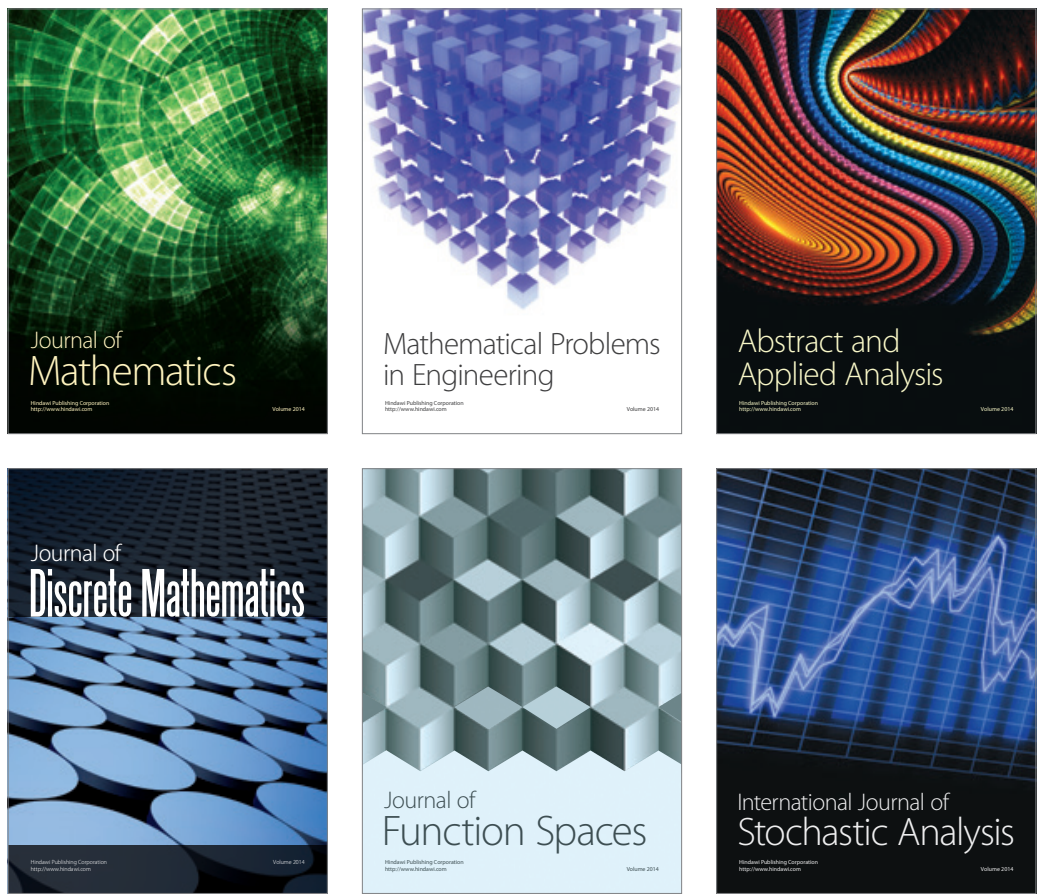

Journal of

Function Spaces

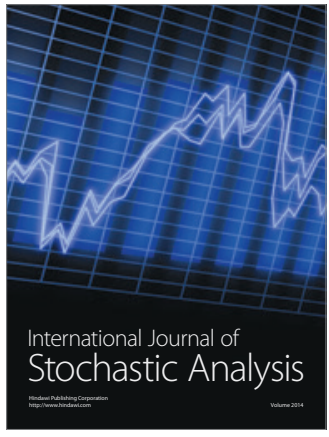

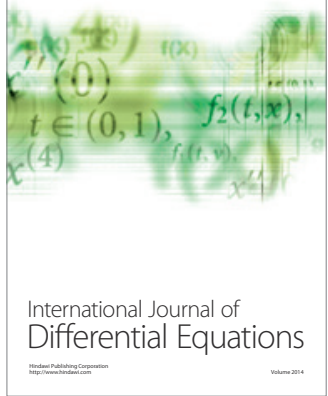
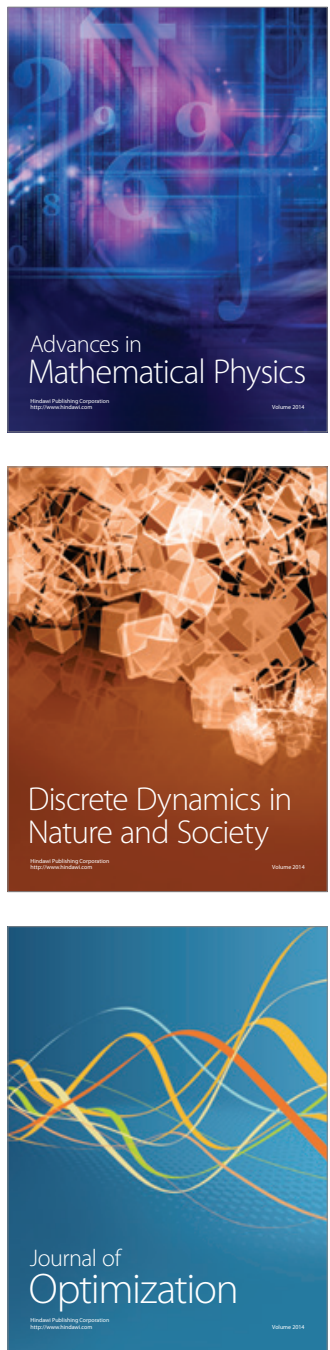